\title{
Разработка альтернативной терминологии надежности
}

Николай И. Плотников, Научно-исследовательский проектный институт гражданской авиации «АвиаМенеджер», Российская федерация, Новосибирск am@aviam.org

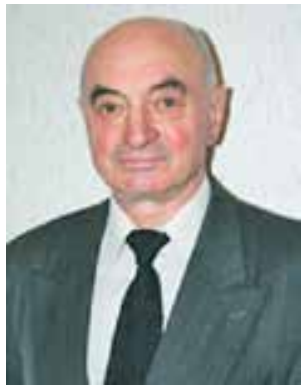

Николай И. Плотников
Резюме. Цель. В настоящей работе составлен краткий исторический обзор эволюции теории надежности техники. Эволюция понятия надежности отображает неразрешенную проблему раскрытия объема и содержания понятия в теории надежности технических объектов. Предлагается логическая проработка и вывод терминов на основании псевдофизической логики высказываний. Представлен подход к решению задачи введением понятия назначения объекта и вывод альтернативных базовых определений надежности. Целью работы является исследование возможности переноса современной теории надежности техники для последующих теоретических разработок и практического применения концепции надежности организаций, социальных групп и человека. Методы. Проблема терминологии и многолетнего поиска определений надежности состоит в дефиците гуманитарной проработки исследуемого предмета: филологической, философской, логической. Безусловно, данные исследования должны выполняться специалистами соответствующих наук. Выполним самостоятельную попытку по теме настоящей работы. Автор предлагает структурный подход исследования терминов. Сущность подхода состоит в следующем. Если выявление признаков содержания понятия затруднительно, возможно осуществить структурирование объема понятия. Структурирование осуществляется через универсальные базы наблюдения: время, пространство, группы и их комбинации: времяпространство, время-группа, пространство-группа. Для этого необходима специальная совокупность терминов. Вводится категория «назначение» как свойство объекта. Понятие назначения обладает большим объемом, является более абстрактным, чем понятие надежности. Отметим, что стандарты качества разрабатывались в концепции назначения как соответствие характеристик объекта требованиям. Отечественные стандарты отдавали предпочтение концепции надежности, где нормативные описания, выдвигаемые определения, такие как «способность выполнять требуемые (заданные) функции, способность (объекта) функционировать», «функционировать, как и когда требуется», «функциональная надежность», «параметрическая надежность», «требования, установленные документацией» просто обобщаются категорией назначения. Данные описания есть не что иное, как указание на свойство назначения объекта. Например, способность объекта перемещаться в пространстве - это свойство назначения, а не надежности. Таким образом, все терминологические поиски стандартизации надежности демонстрируют необоснованное сведение понятия назначения к понятию надежности. Введение категории назначения решает проблемы терминологии в теории надежности. Автор предлагает следующее определение понятия назначения. Назначение - свойство объекта, обусловленное естественным происхождением существования или создаваемого проектируемого применения. Надежность - совокупность состояний как мера соответствия назначению объекта. Заключение. Эволюция содержания понятия надежности техники отображает неразрешенную проблему терминологии в теории надежности технических объектов. Проблема терминологии в значительной степени состоит в нечетком использовании и смешении онтологических терминов. Вывод данных терминов на основании псевдофизической логики и введение категории назначения объекта является основным результатом настоящей работы для введения альтернативной непротиворечивой структуры и содержания терминов надежности. Предлагаемый подход рекомендуется использовать для переработки существующих стандартов.

Ключевые слова: надежность, терминология, онтологические термины, свойство, состояние, событие.

Для цитирования: Плотников Н.И. Разработка альтернативной терминологии надежности // Надежность. 2020. №3. C. 21-26. https://doi.org/10.21683/1729-2646-2020-20-3-21-26

Поступила 25.10.2019 г. / После доработки 15.05.2020 г. / К печати 21.09.2020 г. 


\section{1. Введение}

Нормативные описания надежности разработаны для техники, технических изделий, машин и устройств. Исследования надежности связываются с теорией безопасности и рисков жизнедеятельности. В настоящей работе исследуется проблема терминологии в стандартизации надежности. Целью работы является исследование возможности переноса современной теории надежности техники для последующих теоретических разработок и практического применения концепции надежности организаций, социальных групп и человека.

\section{2. Проблема терминологии теории надежности техники}

Со времени введения понятия надежности техники и появления первых документов с 1950-х годов ведется обсуждение проблем стандартизации терминологии в области надежности, особенно относительно установления основного термина «надежность». Нормативная база включает отечественные и межгосударственные стандарты: ГОСТ 13377-67; ГОСТ 13.337-75; ГОСТ 27 002-83; ГОСТ 27.002-89; ГОСТ 27.002-2015; ГОСТ Р 51901.3-2007 (МЭК 60300-2: 2004); ГОСТ ISO 9000-2011; ГОСТ Р 27.002-2009. Понимание связи надежности рассматривается значительно шире в стандартах, относящихся к терминологии качества, риска, безопасности: ГОСТ 15467-79; ГОСТ Р 51901-2002; ГОСТ Р 51897-2002; ГОСТ Р 51898-2002; ГОСТ 51901.14-2005 (МЭК 61025: 1990); ГОСТ Р 51901.12-2007; ГОСТ Р 53480-2009; ГОСТ Р ИСО/МЭК 31010-2011.

Смысловые описания понятия надежности в документах возможно свести к следующему: «... свойство системы выполнять задание, ...заданные функции, ... установленные показатели в заданных пределах, ... параметров, характеризующих способность выполнять требуемые (заданные) функции...». Сюда же можно отнести смысловое описание в стандартах МЭК по надежности (dependability): «способность (объекта) функционировать как и когда требуется» (ability (of an item) to perform as and when required).

Исходным описанием, раскрывающим содержание и объем понятия надежности, является понятие исправности технического устройства. Поскольку машины работают и с частичными неисправностями, это создает нечеткость в области определения [исправность, неисправность]. Введение термина «отказ» определяет невозможность использования технического изделия. Отсюда возникло определение надежности понятием безотказности и дополнительно: долговечности, ремонтопригодности, сохраняемости. Следующим введением является понятие работоспособности. ГОСТ Р 27.002-2009 надежность определяет понятием готовности, ГОСТ 27.002-2015 [3] - понятием способности. Таким образом, эволюция терминологии за период с 1950-х годов до настоящего времени имеет примерно следующую последовательность замены понятия надежности другими обобщающими «объясняющими» понятиями:

\section{НАДЕЖНОСТЬ $\cong$}

$<$ (исправность $) \rightarrow$ (безотказность $) \rightarrow$ (работоспособность $) \rightarrow$ (готовность $) \rightarrow$ (способность $)>$.

Подробный состав и исторический анализ нормативных документов по терминологии надежности представлен авторами работ $[1,2]$. По мнению автора настоящей работы, введение стандарта [3] не разрешает проблемы стандартизации терминологии надежности. Рассмотрим базовые определения этого стандарта.

Раздел 3 «Термины и определения», пункт 3.1 «Основные понятия»: «3.1.5. надежность: Свойство объекта сохранять во времени способность выполнять требуемые функции в заданных режимах и условиях применения, технического обслуживания, хранения и транспортирования. Прим. 2. Надежность является комплексным свойством, которое в зависимости от назначения объекта и условий его применения может включать в себя безотказность, ремонтопригодность, восстанавливаемость, долговечность, сохраняемость, готовность или определенные сочетания этих свойств». Ниже в стандарте даны определения указанных этих свойств. В п. 3.2 «Состояния» вводятся определения: «3.2.1 исправное состояние (исправность): ..., 3.2 .2 неисправное состояние (неисправность): ...». То есть, указанные свойства рассматриваются и как состояния.

В п. 3.4 «Отказы, дефекты, повреждения» термины «отказ» и «повреждение» определяются как события, а «дефект» - как несоответствие объекта требованиям, установленным документацией. «Причина отказа вызывается», а «последствия отказа обусловливаются» в одновременной и совместной совокупности: явления, процессы, события и состояния. В П. 3.5.5 восстановление: рассматривается и как процесс, и как событие. Подобных особенностей в стандарте множество.

Понятие надежность, без сомнения, является абстрактным, то есть, категорией. Именно это обстоятельство является основной причиной проблемы терминологии и постоянной необходимости раскрывать понятие надежности через другие понятия. Дело усугубляется тем, что используются сложносоставные слова, такие как безотказность, ремонтопригодность, долговечность, восстанавливаемость и другие, которые еще более затрудняют разрешение проблемы. В логике известно, что определение понятия, которое ведет к термину, осуществляется по содержанию (признакам), а не по объему понятия. Если выявление признаков удается, то определение считается сильным, количественным, пригодным для вычислений свойств объектов. В противном случае, определение сводится к простой замене другим понятием или несколькими понятиями («надежность - это безотказность» и т. п.). То есть, определение 
термина является описательным, слабым и непригодным для количественного исчисления. По нашему мнению, выявление признаков содержания понятия надежности непосредственно, «напрямую» не представляется возможным.

Проблема терминологии и многолетнего поиска определений надежности состоит в дефиците гуманитарной проработки исследуемого предмета: филологической, философской, логической. Безусловно, данные исследования должны выполняться специалистами соответствующих наук. Выполним самостоятельную попытку по теме настоящей работы.

\section{3. Исследование терминологии надежности}

Филологический аспект. Филологическое рассмотрение понятия надежности возможно в словарном и лексико-грамматическом аспектах. Словарные поиски слова «надежность» в технической литературе предпринимаются нередко и являются малопродуктивными. Как правило, определения являются описательными и повторяют содержание, установленное в технических документах. По энциклопедическим, специальным, толковым словарям возможно как-то раскрыть объем понятий, но выявление признаков содержания, что необходимо для вывода терминов, является безуспешным.

В лексико-грамматическом аспекте морфологическая структура слова надежность состоит из префикса -на-, корня-морфемы -деж-, и суффикса -ность- (или двух суффиксов -н- и -ость-). Лексико-грамматический разряд слова надежность является суффиксальным отвлеченным (абстрактным) существительным, называющим качество (свойство) или состояние предмета, мотивированное прилагательным надежный со значением отвлеченного признака. «Существительные с суффиксом, представленным морфом -ность-, со значением «носитель признака» называют отвлеченное состояние $[4$, c. 164$]$; ... со зо знчением отвлеченного признака, называют состояние с отвлеченным значением признака, свойства» [4, с. 177]. Теоретически установлено, что существительные мотивируются (управляются) прилагательными в парах: «надежный - надежность». Из данного контекста и хрестоматийного цитирования невозможно установить, что именно называет существительное «надежность» и прилагательное «надежный»свойство, состояние, признак предмета. Таким образом, попытки филологических поисков также не продвигают к решению задачи.

Анализ онтологических терминов в логике. Рассмотрим, как устанавливаются термины, определения и сводимость терминов в построении в логике. Отвлеченные понятия (категории) иначе называют онтологическими терминами (время, пространство, начало, конец, причина), которые затем уточняются (определяются) терминами логики. Из теории понятия, раздела клас- сической логики, известно, что объем и содержание категорий раскрываются наиболее трудно и сложно. Более того, самые абстрактные категории, такие как сущность, вещь, качество и подобные вообще не поддаются обобщению по объему и выявлению признаков по содержанию.

Сначала устанавливают термин (объект). Затем устанавливают предикат (признак). Между термином и предикатом существуют отношения. «Предикат «красный» (и соответствующий «красности» признак) является одноместным - одинарным. Предикат «больше» (и признак величины «больше») является двуместным - бинарным. Предикат с отношением $\geq 2$ является энместным - энарным. Одноместные признаки называют свойствами. Энместные признаки называют отношениями» [5, с. 61]. То есть, высказываниями с многоместными предикатами. Сведение терминов к простым является важнейшей задачей логического анализа научных знаний. «Определить термин - установить его значение, используя другие термины, значение которых уже известно» [5, с. 228]. Термины в построении разделяются на исходные и производные.

D-1. Термин $t^{1}$ является исходным относительно термина $t^{2}$, а $t^{2}$, является производным относительно $t^{1}$ если и только если $t^{1}$ используется при образовании (при установлении значения) $t^{2} \gg[5$, с. 62].

Образование (введение в использование) и определение онтологических терминов является важнейшей частью задачи настоящей работы относительно терминов «свойство», «состояние», «событие», «ситуация», «процесс».

Категории «свойство» и «состояние». Определения понятий «свойство» и «состояние» в стандартах по надежности техники отсутствуют. Между тем, различения данных понятий нетривиальны. По Аристотелю, «обладанием или свойством (hexis) называется проявление некоторой деятельности того, что обладает, и того, чем оно обладает; такое расположение по отношению к другому, например, здоровье есть некоторое свойство»..., «преходящим свойством или состоянием (pathos) называется свойство, по отношению которого возможны изменения; разного рода проявления этих свойств и применения их» [4, с. 244]. Свойство есть качество, состояние есть количество.

Примеры. (1) Тело обладает свойством тяжести и может пребывать в состояниях движения и покоя. (2) Свойство воды проявляется в состояниях жидком, твердом, газообразном (парообразном), кристаллическом. Возможно сказать, что вода обладает «комплексным свойством» и проявляет себя в «подсвойствах» жидкости, льда, пара, снега. Однако, объяснение состояний предпочтительнее.

Таким образом, в существующих стандартах определения надежности как свойства объекта - вопрос дискуссионного выбора. С нашей точки зрения, понятие надежности является состоянием объекта. Обоснования данного выбора и утверждения изложены ниже. 
Категории «событие», «процесс». В стандарте [8] введены следующие определения:

113-01-04 событие (event): То, что имеет место, происходит, наступает в произвольной точке пространства - времени;

113-01-06 процесс (process): Последовательность во времени взаимосвязанных событий.

Данные определения не удовлетворяют поисковым целям настоящей работы, поскольку являются описательными, слабыми. В научной литературе определения рассматриваемых терминов нередко отсутствуют, не сводимы друг другу или отождествляются. Достаточно заметить, что в книге Д.А. Поспелова [9] «Ситуационное управление» определение понятия «ситуация» отсутствует. По А.А. Зиновьеву, «Если Х есть высказывание, то $\downarrow \mathrm{X}$ есть термин события (или состояния). События существуют или не существуют в некоторой данной или любой ситуации» $[5$, с. 166$]$. Здесь, налицо отождествление терминов «событие» и «состояние» и поясняется, что ситуация задается путем указания следующего: а) пространственная область, б) время, в) событие или множество событий, г) комбинирование (a, $\sigma$, в) явно; или ясна из контекста. Однако, из изложенного логического построения непосредственно не выводятся определения терминов «событие», «ситуация» и другие. По нашему мнению, определения могут быть выведены только псевдофизическим (псевдологическим) путем.

Вывод терминов псевдофизической логики. Псевдофизическая логика (ПЛ) (термин Д.А. Поспелова [9]) отношений времени, пространства, причинности и их сочетаний позволяет сформулировать определения, необходимые для последующего изложения. Пусть $\left|O_{i}\right|-$ термин, наблюдаемый объект. Представляем следующие определения и примеры действительности.

D-2. Переходы в пространствах отображения объекта называются восходящими, если осуществляются в направлении большей мерности: $O_{i}: s_{i} s_{i+1}, \ldots, s_{i+k}, i \in \overline{1, n}$, где $n$ - число состояний объекта.

Пример: момент отрыва на взлете летательного аппарата (ЛА) и его переход к движению в трехмерном пространстве.

D-3. Переходы в пространствах отображения объекта называются нисходящими, если осуществляются в направлении меньшей мерности: $O_{i}: s_{i} s_{i-1}, \ldots, s_{i-k}$, $k \in \overline{1, n-1}$ число переходов.

Пример: момент приземления на посадке ЛА и его переход к движению в двухмерном пространстве.

D-4. Переходы в пространствах отображения объектов называются симметричными, если осуществляются в любом их двух направлений одной (большей или меньшей) мерности: $O_{i}: s_{i} s_{i \pm 1}, \ldots, s_{i \pm k}$.

Пример: а) момент разбега ЛА на скорости принятия решения о продолжении или прерывании взлета; б) момент принятия решения на высоте принятия решения (ВПР) о посадке или уходе на второй круг.

D-5. Отображения объектов в восходящих переходах называются разверткой данных описания объекта.
D-6. Отображения объектов в нисходящих переходах называются сверткой данных описания объекта.

Вывод определений терминов «событие», «ситуация» возможен только на соединении временной и пространственной логики. Полный вывод онтологических терминов возможен только на связи временной, пространственной и следственно-причинной (каузальной) логики, что требует дополнительных исследований.

D-7. Отображение объекта в переходах от пространства к пространству $s_{i}$ в момент времени $t_{i}$ называем событием: $e_{i}:\left(t_{i} s_{i} s_{i \pm 1}, \ldots, s_{i \pm k}\right)$, где $e_{i}-$ оператор отображения объекта при переходе от пространства к пространству.

Событие, множество $E, e_{i} \in E$, является связью ПЛ отношений времени и пространства $e_{i}\left(t_{i}, s_{i}\right)$.

D-8. Совокупность связей ПЛ отношений времени и пространства называем ситуацией.

В вышеизложенных определениях и примерах: ситуация - совокупность (начало движения и остановки объекта, взлеты и посадки, принятия решений) событий в восходящих, нисходящих, симметричных переходах.

В стандарте [8] определение понятия «состояние» отсутствует. Вводим следующее определение.

D-9. Состояние - параметр, совокупность параметров свойств объекта в наблюдаемый интервал времени («113-01-10 интервал времени (time interval): Часть оси времени, ограниченной двумя мгновениями» [8]).

В данном определении понятие состояния определяется как параметр соизмерения показателей (совместного и одновременного). Например, скорость транспортного средства соразмеряется двумя показателями: расстояния и времени.

\section{4. Решение задачи разработки терминологии надежности}

Структурный подход. Автор предлагает структурный подход исследования терминов. Сущность подхода состоит в следующем. Если выявление признаков содержания понятия затруднительно, возможно осуществить структурирование объема понятия. Структурирование осуществляется через универсальные базы наблюдения: время, пространство, группы и их комбинации: время-пространство, время-группа, пространство-группа [6]. Эмпирически установлено, что надежность объекта любой природы изменяется во времени по этапам, так называемого, U-образного профиля: вход (ниже нормы), нормализация, старение (ниже нормы). Следовательно, надежность любого объекта можно достоверно наблюдать (измерять, оценивать) по U-профилю. Для этого необходима специальная совокупность терминов.

Предлагаем строгую иерархическую структуру терминов. Объект имеет наименование (name). Назначение (appointment) объекта называет (устанавливает) его свойство (property). Свойство наблюдается (измеряется, оценивается) в состояниях (state) или (параметрах) 
(parameter). Состояния образуются совокупностью показателей (indicator). Показатели устанавливаются по значениям (value). Для вычислений свойств объекта устанавливается информационная единица (ИЕ) энарной пятеркой:

$\{1-$ назначение $\subseteq 2-$ свойство $\subseteq 3-$ состояние (параметр) $\subseteq 4-$ показатель $\subseteq 5$ - значение $\}$.

D-10. ИЕ является точной (точечной), если подмножество, соответствующее значению, является одноточечным и его нельзя разбить на части.

D-11. Точность связана со значением.

Каждый элемент ИЕ наблюдается (выявляется, определяется) по признакам (sign). ИЕ имеет иерархию, исключает нечеткое толкование и произвольное применение терминов. Представленный комплекс терминов является универсальной моделью, может применяться для вычислений состояний объектов любой природы. Структура терминов представлена на схеме в работе $[10$, c. 91$]$.
Структурный подход предполагает также поиск и установление обобщающего, более абстрактного понятия, по отношению к исследуемому. По нашему мнению, подобным понятием является категория назначения. Основанием для данного утверждения является следующее.

Категория «назначение» как свойство объекта. Понятие назначения обладает большим объемом, является более абстрактным, чем понятие надежности. Отметим, что стандарты качества (ISO) разрабатывались в концепции назначения как соответствие характеристик объекта требованиям (ГОСТ ISO 9000-2011: «Качество: степень соответствия совокупности и присущих характеристик требованиям»).

Отечественные стандарты отдавали предпочтение концепции надежности, где нормативные описания, выдвигаемые определения, такие как «способность выполнять требуемые (заданные) функции, способность (объекта) функционировать», «функционировать, как и

Табл. 1. Образец разработки альтернативной терминологии теории надежности техники

\begin{tabular}{|c|c|}
\hline ГОСТ 27.002-2015 & Альтернативные определения \\
\hline 3.2 Состояния & Состояния надежности \\
\hline $\begin{array}{l}3.2 .1 \text { исправное состояние (исправность): Состояние объекта, в } \\
\text { котором он соответствует всем требованиям, установленным в } \\
\text { документации на него }\end{array}$ & $\begin{array}{c}\text { Исправное состояние: состояние, соответству- } \\
\text { ющее назначению объекта при наличии допусти- } \\
\text { мых повреждений. }\end{array}$ \\
\hline $\begin{array}{c}3.2 .2 \text { неисправное состояние (неисправность): Состояние объек- } \\
\text { та, в котором он не соответствует хотя бы одному из требований, } \\
\text { установленных в документации на него }\end{array}$ & $\begin{array}{l}\text { Неисправное состояние: состояние, не соответ- } \\
\text { ствующее назначению объекта из-за дефектов. }\end{array}$ \\
\hline $\begin{array}{c}\text { 3.2.3 работоспособное состояние: Состояние объекта, в котором } \\
\text { он способен выполнять требуемые функции }\end{array}$ & $\begin{array}{l}\text { Работоспособное состояние: состояние, соот- } \\
\text { ветствующее назначению объекта. }\end{array}$ \\
\hline $\begin{array}{c}3.2 .4 \text { неработоспособное состояние: Состояние объекта, в кото- } \\
\text { ром он не способен выполнять хотя бы одну требуемую функцию } \\
\text { по причинам, зависящим от него или из-за профилактического } \\
\text { технического обслуживания }\end{array}$ & $\begin{array}{l}\text { Неработоспособное состояние: состояние, не } \\
\text { соответствующее назначению объекта. }\end{array}$ \\
\hline 3.4 Отказы, дефекты, повреждения & События нарушения надежности \\
\hline $\begin{array}{c}3.4 .1 \text { отказ: Событие, заключающееся в нарушении работоспособ } \\
\text { ного состояния объекта }\end{array}$ & $\begin{array}{l}\text { Отказ: событие нарушения работоспособного } \\
\text { состояния объекта. }\end{array}$ \\
\hline $\begin{array}{c}3.4 .2 \text { дефект: Каждое отдельное несоответствие объекта требова- } \\
\text { ниям, установленным документацией }\end{array}$ & $\begin{array}{c}\text { Дефект: событие нарушения исправного состоя- } \\
\text { ния объекта. }\end{array}$ \\
\hline $\begin{array}{c}\text { 3.4.3 повреждение: Событие, заключающееся в нарушении ис- } \\
\text { правного состояния объекта при сохранении работоспособного } \\
\text { состояния }\end{array}$ & $\begin{array}{c}\text { Повреждение: событие нарушения исправного } \\
\text { состояния объекта при сохранении работоспо- } \\
\text { собного состояния }\end{array}$ \\
\hline 3.5 Техническое обслуживание, восстановление и ремонт & Процессы восстановления надежности \\
\hline $\begin{array}{l}\text { 3.5.2 техническое обслуживание; ТО: Комплекс организационных } \\
\text { мероприятий и технических операций, направленных на под- } \\
\text { держание работоспособности (исправности) объекта и снижение } \\
\text { вероятности его отказов при использовании по назначению, хра- } \\
\text { нении и транспортировании. } \\
\end{array}$ & $\begin{array}{l}\text { Техническое обслуживание; ТО: процесс, на- } \\
\text { правленный на сохранение работоспособного и } \\
\text { исправного состояний объекта. }\end{array}$ \\
\hline $\begin{array}{l}3.5 .5 \text { восстановление: Процесс и событие, заключающиеся в пере } \\
\text { ходе объекта из неработоспособного состояния в работоспособ- } \\
\text { ное. }\end{array}$ & $\begin{array}{c}\text { Восстановление: процесс, направленный на пе- } \\
\text { реход объекта из неработоспособного состояния } \\
\text { в работоспособное состояние. }\end{array}$ \\
\hline $\begin{array}{c}\text { 3.5.9 ремонт: Комплекс технических операций и организацион- } \\
\text { ных действий по восстановлению исправного или работоспособ- } \\
\text { ного состояния объекта и восстановлению ресурса объекта или } \\
\text { его составных частей. }\end{array}$ & $\begin{array}{l}\text { Ремонт: процесс, направленный на переход объ- } \\
\text { екта из неисправного состояния в работоспособ- } \\
\text { ное состояние. }\end{array}$ \\
\hline
\end{tabular}


когда требуется», «функциональная надежность», «параметрическая надежность», «требования, установленные документацией» просто обобщаются категорией назначения. Данные описания есть не что иное, как указание на свойство назначения объекта. Например, способность объекта перемещаться в пространстве - это свойство назначения, а не надежности.

Если автомобиль «стоит в пробке» (пример, который приводят авторы работы [2]), не предназначен быть еще и вертолетом, или самолетом как в фильме «Фантомас», это заложено в проекте его назначения - перемещение в двухмерном пространстве. Поэтому абсолютно надежный автомобиль будет стоять в пробке «надежно» и неподвижно.

Таким образом, все терминологические поиски стандартизации надежности демонстрируют необоснованное сведение понятия назначения к понятию надежности. Введение категории назначения решает проблемы терминологии в теории надежности. Автор предлагает следующее определение понятия назначения.

D-12. Назначение - свойство объекта, обусловленное естественным происхождением существования или создаваемого проектируемого применения.

Свойство естественного происхождения: назначение «щуки в озере, чтоб карась не дремал».

Данное определение вводится для простого объекта. Назначение сложного объекта допустимо рассматривать из элементов свойств. Если принять выдвигаемую точку зрения, что понятие назначения является свойством, то все производные понятия являются состояниями. Подобными состояниями в контексте настоящей работы принимаются: надежность, безопасность (защищенность), риск, эффективность и другие.

Вывод альтернативной терминологии теории надежности техники. Исходя из вышеизложенного содержания представим пример (образец) разработки альтернативной терминологии теории надежности. Предлагаются только базовые определения. Полная переработка стандарта не входит в задачу настоящей работы.

D-13. Надежность - совокупность состояний как мера соответствия назначению объекта. Альтернативные определения изложены в следующем содержании (табл. 1).

Образец разработки терминологии исключает тождественное использование терминов типа «исправное состояние (исправность)». Полагаем, что термины событий и процессов должны быть подвергнуты тщательному исследованию в филологическом и логическом аспектах для установления четкой различимости. Так вместо термина «дефект» возможно использование термина «поломка» и т.п.

\section{5. Заключение}

Эволюция содержания понятия надежности техники отображает неразрешенную проблему терминологии в теории надежности технических объектов. Проблема терминологии в значительной степени состоит в нечетком использовании и смешении онтологических терминов. Вывод данных терминов на основании псевдофизической логики и введение категории назначения объекта является основным результатом настоящей работы для введения альтернативной непротиворечивой структуры и содержания терминов надежности. Предлагаемый подход рекомендуется использовать для переработки существующих стандартов.

\section{Библиографический список}

1. Нетес В.А., Тарасьев Ю.И., Шпер В.Л. Актуальные вопросы стандартизации терминологии в области надёжности // Надежность. 2014. № 2. С. 116-119.

2. Нетес В.А., Тарасьев Ю.И., Шпер В.Л. Как нам определить что такое «надёжность» // Надежность. 2014. № 4. С. 3-14.

3. ГОСТ 27.002-2015. Надежность в технике. Термины и определения. М.: Стандартинформ, 2016. 24 с.

4. Русская грамматика. М.: Наука, 1980. Т. 1. 784 с. T. $2.710 \mathrm{c}$.

5. Зиновьев А.А. Логика науки. М.: Мысль, 1971. $279 \mathrm{c}$.

6. Аристотель. Политика. Метафизика. Аналитика [пер с древнегреческого]. М.: Эксмо; СПб.: Мидгард, 2008.960 c.

7. Клир Джордж. Системология. Автоматизация решения системных задач М.: Радио и связь, 1990. 544 с.

8. ГОСТ IEC 60050-113-2015. Международный электротехнический словарь. Часть 113. Физика в электротехнике. М.: Стандартинформ, 2016. 51 с.

9. Поспелов Д.А. Ситуационное управление. М.: Наука, 1986. 286 с.

10. Плотников Н.И. Основания теории надежности человека-оператора (пилота) // Надежность. 2015. № 2 (53). С. 90-93.

\section{Сведения об авторе}

Плотников Николай Иванович - кандидат технических наук, генеральный директор Научно-исследовательского проектного института гражданской авиации «АвиаМенеджер», Российская федерация, Новосибирск, e-mail: am@aviam.org

\section{Вклад автора в статью}

Автором выполнен анализ стандартов надежности техники. Выполнен словарный, лексико-грамматический и логический анализ понятия надежность. Предложено новое альтернативное определение понятия надежности и вариант переработки стандарта.

\section{Конфликт интересов}

Автор заявляет об отсутствии конфликта интересов. 